\title{
Modulation of microglia by Wolfberry on the survival of retinal ganglion cells in a rat ocular hypertension model
}

\author{
Kin Chiu • Hiu-Chi Chan • Sze-Chun Yeung • \\ Wai-Hung Yuen • Sze-Yong Zee • \\ Raymond Chuen-Chung Chang • Kwok-Fai So
}

Received: 5 February 2009 /Accepted: 2 June 2009/Published online: 1 July 2009

(C) The Author(s) 2009. This article is published with open access at Springerlink.com

\begin{abstract}
The active component of Wolfberry (Lycium barbarum), lycium barbarum polysaccharides (LBP), has been shown to be neuroprotective to retinal ganglion cells (RGCs) against ocular hypertension (OH). Aiming to study whether this neuroprotection is mediated via modulating immune cells in the retina, we used multiphoton confocal microscopy to investigate morphological changes of microglia in whole-mounted retinas. Retinas under $\mathrm{OH}$ displayed slightly activated microglia. One to $100 \mathrm{mg} / \mathrm{kg}$ LBP exerted
\end{abstract}

K. Chiu $\cdot$ S.-C. Yeung $\cdot$ R. C.-C. Chang $(\bowtie) \cdot$ K.-F. So

Laboratory of Neurodegenerative Diseases,

Department of Anatomy, LKS Faculty of Medicine,

The University of Hong Kong,

Pokfulam, Hong Kong SAR, China

e-mail: rccchang@hkucc.hku.hk

K. Chiu $\cdot$ H.-C. Chan $\cdot$ R. C.-C. Chang $\cdot$ K.-F. So

State Key Laboratory of Brain and Cognitive Sciences,

The University of Hong Kong,

Pokfulam, Hong Kong SAR, China

K. Chiu $\cdot$ R. C.-C. Chang $\cdot$ K.-F. So

Research Centre of Heart, Brain, Hormone and Healthy Aging,

LKS Faculty of Medicine, The University of Hong Kong,

Pokfulam, Hong Kong SAR, China

W.-H. Yuen

Department of Chemistry, The University of Hong Kong,

Pokfulam, Hong Kong SAR, China

S.-Y. Zee

United College, Hong Kong Baptist University,

Kowloon, Hong Kong SAR, China

K.-F. So $(\bowtie)$

Laboratory Block, Faculty of Medicine Building,

Department of Anatomy, The University of Hong Kong,

Rm. L1-49, 21 Sassoon Road,

Pokfulam, Hong Kong SAR, China

e-mail: hrmaskf@hkucc.hku.hk the best neuroprotection and elicited moderately activated microglia in the inner retina with ramified appearance but thicker and focally enlarged processes. Intravitreous injection of lipopolysaccharide decreased the survival of RGCs at 4 weeks, and the activated microglia exhibited amoeboid appearance as fully activated phenotype. When activation of microglia was attenuated by intravitreous injection of macrophage/microglia inhibitory factor, protective effect of $10 \mathrm{mg} / \mathrm{kg}$ LBP was attenuated. The results implicated that neuroprotective effects of LBP were partly due to modulating the activation of microglia.

Keywords Wolfberry - Glaucoma Microglia .

Neuroprotection

\section{Introduction}

Wolfberry (fruit of Lycium barbarum Linn, in the family Solanaceae) is known as Fructus Lycii and L. barbarum in the West and Gouqizi or Kei Tze in Asia. In traditional Chinese medicine literature, it has been known for balancing "Yin" and "Yang" in the body, nourishing the liver and kidney, improving visual acuity for more than 2,500 years [1]. The content of Wolfberry contains about $40 \%$ polysaccharides [lycium barbarum polysaccharides (LBP)]; therefore, research in Wolfberry often focuses on these water-soluble fractions. LBP as food supplement enhances the body defense system by restoring atrophied thymus in aged subjects and regulates the proliferation and the immune activity of splenocytes and T cells [2-6]. It has been shown that LBP can increase phagocytic activity of macrophages, immune activity of cytotoxic $\mathrm{T}$ cells, and natural killer (NK) cells in cyclophosphamide-treated and S180-bearing mice [7-9]. LBP increases interleukin-2 
(IL-2) receptors on isolated human peripheral lymphocytes [10]. LBP can be purified into different fractions; glycoconjugate $\mathrm{LBP}_{3 \mathrm{P}}$ can increase the expression of messenger RNA and protein level of IL-2 and tumor necrosis factor- $\alpha$ (TNF- $\alpha$ ) in human peripheral blood mononuclear cells [2] and increase phagocytosis by macrophage, antibodies secreted by spleen cells, spleen lymphocyte proliferation, and cytotoxic T cells activity in S180-bearing mice [9]. Our previous study reported the neuroprotective effects of LBP on RGCs in an experimental model of glaucoma [11]. However, it is unclear whether neuroprotection is mediated via modulating immune cells in the retina, which is our aim in this study.

Increasing lines of evidence obtained from clinical and experimental studies strongly suggests an aberrant activity of the immune system in glaucoma [12, 13]. Microglial cells are the major immunocompetent cells in the central nervous system (CNS). It has been reported that microglia have diverse phenotypes, which secrete beneficial or destructive factors [14]. Activated microglia have been considered to be endogenous malefactors in the CNS; they induce neuronal death by releasing excess cytotoxic factors such as superoxide [15], nitric oxide, and TNF- $\alpha$ [16-18]. However, increasing lines of evidence have shown that the protective effects of microglia can be accomplished by releasing trophic and anti-inflammatory factors [19-24]. Whether microglia exhibit neuroprotective or neurodestructive effects depends on the disease state or the type of stimulus. There are increasing lines of evidence in vitro, showing that it is possible to manipulate the activation state of microglia so that their activation can be beneficial, i.e., protecting rather than destroying neurons [25]. However, it is difficult to achieve this goal in vivo, especially in a chronic neurodegenerative model.

A primary objective in this study is to evaluate the modulation of LBP on retinal microglia and its neuroprotective effect on survival of RGCs in a chronic ocular hypertension $(\mathrm{OH})$ model. We studied the morphology of microglia in $\mathrm{OH}$ retina from rats fed with different doses of LBP. In addition, the effect on the survival of RGCs after administration of either a microglia activation inhibitor, macrophage/microglia inhibitory factor (MIF), or microglia activation stimulator, LPS (bacterial endotoxin lipopolysaccharide) was evaluated in $\mathrm{OH}$ rats.

\section{Materials and methods}

\section{Preparation of LBP}

The Wolfberry originated from NingXia Huizu Autonomous Region, the People's Republic of China. The simplified extraction scheme of LBP from Wolfberry [26] has been reported by our group. Briefly, the dried wolfberries $(10 \mathrm{~kg})$ were grounded to fine powder and defatted by refluxing with 95\% ethanol. The insoluble residue was filtered, airdried, and extracted successively with $70^{\circ} \mathrm{C}$ hot water. The concentrated extract was incubated with trichloroacetic acid, extensively dialyzed against running distilled water, concentrated, and then precipitated using 95\% ethanol. After centrifugation and several rinses with absolute ethanol and acetone, the resulting precipitate was vacuum dried at $40^{\circ} \mathrm{C}$ to yield a brown powder Wolfberry extract-LBP (2 g).

\section{Animal grouping}

Sixty-six adult female Sprague-Dawley rats (250-280 g) were obtained from the Laboratory Animal Unit of the LKS Faculty of medicine in the University of Hong Kong and were maintained in a temperature-controlled room with a 12-h light/dark cycle throughout the observation period. The animals were handled according to the protocol for the use of animal in research approved by the Committee on the Use of Live Animals in Teaching and Research of the University of Hong Kong and the Association for Research in Vision and Ophthalmology (ARVO, USA) statements for the use of animals in Ophthalmic and Vision Research. Prior to measuring intraocular pressure (IOP) or any other operations, the rats were anesthetized with an intraperitoneal injection of a ketamine/xylazine mixture (ketamine $80 \mathrm{mg} / \mathrm{kg}$ and xylazine $8 \mathrm{mg} / \mathrm{kg}$; Alfasan, Woerden, Holland). Prior to every ocular photocoagulation (including IOP measurement, laser treatment, and intravitreous injection), one drop of proparacaine hydrochloride $(0.5 \%$ alcaine, Alcon-Couvreur, Belgium) was applied to the eyes as a topical anesthetic. After every ocular manipulation, ophthalmic Tobrex ointment (3\% tobramyxin, AlconCouvreur, Belgium) was applied topically on the eyes to prevent infection. All operations were performed under an operating microscope (Olympus OME, Tokyo, Japan).

The animals were divided into 11 groups, and every group consisted of six rats (Table 1). The LBP powder was dissolved in $0.01 \mathrm{M}$ sterilized phosphate-buffered saline (PBS; pH 7.4). Animals were fed daily through a nasogastric tube with $1 \mathrm{ml}$ of either PBS or different dosages of LBP, including 1, 10, 100, 1,000 mg/kg. Daily feeding (groups 2-10) started at 7 days before the first laser treatment and continued until euthanization of the rats. IOP was measured before the first laser treatment (as baseline) and before killing (postoperative). A total of two laser photocoagulation was performed at 1 -week interval.

\section{Ocular hypertension model}

$\mathrm{OH}$ was induced in the right eye of each animal using laser photocoagulation according to our previous publications 
Table 1 Experimental grouping

\begin{tabular}{|c|c|c|c|}
\hline \multirow[t]{2}{*}{ Treatment groups (right eye treated) } & \multicolumn{2}{|c|}{$\begin{array}{l}\text { Survival time after the first laser } \\
\text { photocoagulation }\end{array}$} & \multirow[t]{2}{*}{ Objectives } \\
\hline & 2 weeks & 4 weeks & \\
\hline No laser & 6 (group 1) & & Normal control \\
\hline Laser treatment + fed with $0.01 \mathrm{M} \mathrm{PBS}$ & 6 (group 2) & 6 (group 9) & Solvent control for drug treatment \\
\hline $\begin{array}{l}\text { Laser treatment }+ \text { fed with } 1 \mathrm{mg} / \mathrm{kg} \text { LBP } \\
\text { Laser treatment }+ \text { fed with } 10 \mathrm{mg} / \mathrm{kg} \mathrm{LBP}\end{array}$ & $\begin{array}{l}6(\text { group } 3) \\
6(\text { group } 4)\end{array}$ & 6 (group 10) & LBP feeding dose -response study \\
\hline Laser treatment + fed with $100 \mathrm{mg} / \mathrm{kg} \mathrm{LBP}$ & 6 (group 5) & & \\
\hline Laser treatment + fed with $1000 \mathrm{mg} / \mathrm{kg}$ LBP & 6 (group 6) & & \\
\hline $\begin{array}{l}\text { Laser treatment }+ \text { fed with } 10 \mathrm{mg} / \mathrm{kg} \mathrm{LBP}+\text { i.v. } \\
\text { injection of } 0.01 \mathrm{M} \text { PBS }\end{array}$ & 6 (group 7) & & Inhibition of microglia activation by MIF \\
\hline $\begin{array}{l}\text { Laser treatment }+ \text { fed with } 10 \mathrm{mg} / \mathrm{kg} \text { LBP }+ \text { i.v. } \\
\text { injection of } 172 \mathrm{ng} \text { MIF dissolved in } 0.01 \mathrm{M} \mathrm{PBS}\end{array}$ & 6 (group 8 ) & & \\
\hline Laser treatment + i.v. injection of LPS $(50 \mu \mathrm{g})$ & & $6($ group 11) & Served as microglia activation control \\
\hline
\end{tabular}

$[11,27,28]$. Briefly, the limbal vein and the three radical episcleral aqueous humor drainage veins (superior nasal, superior temporal, and inferior temporal) were photocoagulated using an Argon laser (Ultima 2000SE Argon Laser, Coherent, USA). About 60 laser spots (power, 1,000 mV; spot size, 50-100 $\mu \mathrm{m}$; duration, $0.1 \mathrm{~s}$ ) around the limbal vein (except the nasal area) and 15-20 laser spots on each episcleral aqueous humor drainage vein were applied. To maintain a high IOP, a second laser treatment at the same settings was applied 7 days later.

\section{Measurement of IOP}

IOP was measured with a Tonopen XL tonometer (Mentor ${ }^{\circledR}$, Norwell, USA) before the first laser treatment and every subsequent week until the rats were killed. To avoid diurnal variation and effect of anesthesia, all IOP measurements were taken at 10 A.M. and within 15-30 min after administration of ketamine and xylazine mixture (i.p.). An average of ten measurements was used to determine the IOP of the eye.

Intravitreous injection

Tuftsin fragment 1-3 acetate salt, also known as MIF, was purchased from Sigma (St Louis, MO, USA); bacterial endotoxin lipopolysaccharide (LPS) derived from Escherichia coli O111:B4 was purchased from Calbiochem (La Jolla, CA, USA). To study the influence of MIF on the survival of RGCs and microglia activation, immediately after the first laser treatment, $2 \mu \mathrm{m}$ of $0.01 \mathrm{M}$ PBS (group 7) or $172 \mathrm{ng}$ of MIF (2.5 mM) in PBS (group 8) were injected into the vitreous cavity of the right eye [29] of the $10 \mathrm{mg} / \mathrm{kg}$ LBP-fed group. To demonstrate the fully activated microglia in the retina, $5 \mu \mathrm{g}$ of LPS in $2 \mu \mathrm{l}$ PBS was injected into the vitreous cavity of the right eye (group 11) immediately after the first laser treatment. Animals with cataract, intraocular bleeding, retinal detachment, or non-elevated IOP were excluded from this study ( $\sim 15 \%$ of experimental animals).

\section{Retrograde labeling of RGCs}

To evaluate the drug effects on RGCs, they were retrogradely labeled by applying FG on the surface of superior colliculus (SC) at 4 days prior to euthanization [30]. Briefly, the rat scalp was cut open in the mid-line, and a small hole was drilled on the skull on each side of the sagittal suture. The four edges of SC can be observed directly under the operation microscope after removing the overlying cerebral cortex. Then, a thin layer of gelatin sponge (UpJohn, Kalamazoo, MI, USA) pre-soaked with 6\% FG (Fluorochrome, Denver, CO, USA) was placed on the surface of SC (FG is taken up by the axon terminals of RGCs and bilaterally transported retrogradely to its somata in the retina). Then, the scalp was sutured, and an analgesic, bupreorphine $(100 \mathrm{mg} / \mathrm{kg})$, was orally administered for 5 days for pain relief.

Counting of RGCs and statistical analysis

At different time points, the rats were killed with an overdose of a mixture of ketamine/xylazine after measuring the IOP. Both eyes were enucleated and post-fixed in $4 \%$ paraformaldehyde for $60 \mathrm{~min}$, then cut horizontally into superior and inferior eyecups. The superior eyecups with intact optic nerves were fixed overnight and processed to make paraffin blocks. Retinas from the inferior eyecups were dissected from the underlying sclera, and two cuts were made to divide the retina into three (nasal, inferior, and temporal) quadrants. The dissected retinas were then flattened with the vitreal side up and mounted using 
fluorescent mounting medium (Dako, Carpentaria, CA, USA). The FG-labeled RGCs (FG particles in the cytoplasm) were visualized at $\times 40$ magnification using a fluorescent microscope with a UV-385 filter (Nikon, Kawasaki, Japan). The photos of RGCs were taken (200× $200 \mu \mathrm{m}^{2} /$ microscope field) with each $500-\mu \mathrm{m}$ separation along the median line of each quadrant (eight microscopic fields/quadrant) starting from the optic disc to the peripheral border of the retina. After counting of the RGCs with the aid of a computer software developed by us (manuscript in preparation), the results were manually double checked by a person who was blinded to the grouping. The average density of RGCs was calculated for the entire retina.

To evaluate different effects of various treatments, changes in the density of FG-labeled RGCs were expressed as a percentage loss of FG-labeled RGCs by comparing the laser-treated right eye and normal control eye:

$\left[\frac{\text { Density of RGCs in the normal eye density of RGCs in the right eye with } \mathrm{OH}}{\text { Density of RGCs in the normal eye }}\right] \times 100 \%$

The percentage loss of FG-labeled RGCs in different treatment groups was compared using one-way analysis of variance followed by a post hoc Tukey multiple comparison test (SigmaStat ${ }^{\circledR}$, statistical significance is noted as $p<0.05$ ).

Immunohistochemistry of microglia in retinal sections

Retinal sections from different groups were handled at the same time for each primary antibody to avoid bench to bench variation. Four micrometer cross-retinal sections with intact optic nerves were used to detect OX42 immunoreactivity. The sections were deparaffinized and boiled in citric acid buffer (0.01 M, pH 6.0, $15 \mathrm{~min})$. Catalytic enhancement was performed by incubation in $1 \% \mathrm{H}_{2} \mathrm{O}_{2}$ for $15 \mathrm{~min}$. Following washing and blocking, retinal sections were incubated with mouse anti-rat monocolonal OX42 primary antibody (1:25, Pharmingen, California, USA) overnight at $4^{\circ} \mathrm{C}$. After further washing, retinal sections were incubated with biotinylated goat and mouse secondary antibody (Molecular Probe) at room temperature for $1 \mathrm{~h}$. Then, the sections were washed with TBS and incubated with avidin-biotin complex (Vector Lab, USA) for $1 \mathrm{~h}$. The slides were washed in TBS twice and once in imidozole-acetate buffer $(175 \mathrm{mM}$ acetate, $10 \mathrm{mM}$ imidazole, $\mathrm{pH} 7.2$ ), for $10 \mathrm{~min}$ each. The sections were further incubated with 3,3-diaminobenzidine (DAB; $10 \mathrm{mM}$ $\mathrm{NiSO}_{4}, 125 \mathrm{mM}$ acetate, $10 \mathrm{mM}$ imidozole, $0.03 \%$ DAB, $0.003 \% \mathrm{H}_{2} \mathrm{O}_{2}, \mathrm{pH} 7.2$ ) for $10 \mathrm{~min}$. The sections were mounted with Permount medium. OX42-immunoreactive signals were observed under a light microscope. Images were captured using the Spot-Advance Digital system (Spot RT; Diagnostic Instruments, Sciscope Instrument Companies, USA). The specificity of the antibody was tested by omission of the primary antibody.

Immunohistochemistry of microglia in flat-mounted retina

In the 4-week study (groups 9, 10, and 11), the flat-mounted retinas were carefully removed from the slides and rehydrated in $0.1 \mathrm{M}$ PB using 48-well plate. There were six rats in each group, and three retinas from each group were used for the flat-mounted immunohistochemical study. The retinas were washed with $0.1 \mathrm{M} \mathrm{PB}$ with constant shaking at $4^{\circ} \mathrm{C}$ overnight to wash out the fluorescent mounting medium. Then, they were blocked with $10 \%$ normal goat serum in $0.1 \mathrm{M}$ $\mathrm{PB}$ containing $1 \%$ Triton $\mathrm{X}-100$ for about $2 \mathrm{~h}$ at $4^{\circ} \mathrm{C}$. After three washings of ice-cold $0.1 \mathrm{M} \mathrm{PB}$, the retinas were incubated with ionized calcium adaptive molecular 1 (iba-1) primary antibody (1:800; Wako Chemicals USA, Richmond, USA) for 3 days at $4 \mathrm{C}$. To visualize microglia, the retinas were incubated with Alexa-594 fluorescent-conjugated secondary antibody (1:800; Molecular Probe, USA) for $1 \mathrm{~h}$ at room temperature. To visualize different layers of the retina, they were further incubated with $0.2 \%$ diamidino-2-phenylindole (DAPI) for $1 \mathrm{~h}$ at room temperature. In between each incubation step, there were three turns of $0.1 \mathrm{M} \mathrm{PB}$ washing for $10 \mathrm{~min}$ each. Finally, the retinas were flat-mounted using fluorescent mounting medium with the vitreal side facing upward.

Under fluorescent microscope, the iba-1 signal was checked throughout the retina, and there was no regional difference observed. Therefore, one representative retinal area of $230 \times 230 \mu \mathrm{m}^{2}$ at about $2,500 \mu \mathrm{m}$ from the optic disc of each retina was scanned at $\times 40$ magnification using a LSM-510Meta multiphoton confocal microscope (Carl Zeiss, Jena, Germany). All the images were taken under exactly the same excitation attenuation to avoid bias on the judgment of the immunoreactivity. The gain level for all groups was identical in order to demonstrate the best resolution of the microglia in the normal control group. Guided by the morphology of DAPI-stained nuclei, scanning started from the surface of inner-limiting membrane to the outer nuclear layer. The $\mathrm{Z}$ interval was $1 \mu \mathrm{m}$ among different focal planes, and depending on the retinal thickness, 80 planes would be scanned for each retina. On average, the scanning time for each retina was around $1 \mathrm{~h}$. Vertical figure configured by the LSM software illustrated that most of the microglia were in the inner retina (from 
inner-limiting membrane to the outer most layer of the inner nuclear layer). Stacked images of different focal planes in the inner retina were created using the LSM software in order to display the entire microglia cell body and their processes. To further demonstrate the morphology of different status of microglia, representative single cells were chosen from the $\times 40$ magnification region and then re-scanned under $\times 63$ magnification. The scan started from the upper border of the processes to the soma and then to the lower border of the processes; the Z-interval was also $1 \mu \mathrm{m}$.

\section{Results}

Laser photocoagulation increased the IOP of the right eyes (OH eyes) about 1.7 times compared to the contralateral control eyes (23.4 vs. $13.9 \mathrm{mmHg}$ ). Oral feeding of PBS or various doses of LBP did not alter the level of IOP in all animals.

Monoclonal OX42 antibody that recognizes the expression of complement receptor 3 on the microglia was used to detect all status of microglia (from resting to fully activated status) in the retinal sections. In normal retinas (group 1), only processes-like signals of OX42 without identifiable perikarya or cell body were observed (Fig. 1a). At 2 weeks after the first laser-induced $\mathrm{OH}$, there was a loss of $17 \%$ of RGCs in the PBS-fed rats (group 2) [11]. OX42-positive microglia were observed in the inner retina [including ganglion cell layer (GCL), inner plexiform layer, and inner nuclear layer (INL)] with ramified morphology (Fig. 1b). These cells were slightly activated and exhibited small perikarya with long thin branching processes that were detectable in $4-\mu \mathrm{m}$ sections. We repeated the LBP-fed doses that previously showed significant protection on the survival of RGCs (groups 3, 4, and 5). The loss of RGCs was $1 \%$ in $1 \mathrm{mg} / \mathrm{kg}, 0 \%$ in $10 \mathrm{mg} / \mathrm{kg}$, and $2.4 \%$ in $100 \mathrm{mg} / \mathrm{kg}$ [11]. In LBP-fed rats, both the number and intensity of OX42-positive microglia increased in parallel with the increase in dosages of LBP feeding. In animals with administration of LBP from 1 to $100 \mathrm{mg} / \mathrm{kg}$, the majority of microglia in the retina of groups 3-5 was in ramified morphology (Fig 1c-e). However, the processes became thicker, and the branching increased compared with the PBS group. The maximum radial extent of the cell (soma axis + longest processes) was about $50 \mu \mathrm{m}$. We defined this as a moderate activation status. In $1,000 \mathrm{mg} / \mathrm{kg}$ group (group 6), there was much less protection on RGCs as shown by us previously [11]. In the retinas of this group of animals, most of the OX42 immunoreactive positive microglia was intensely stained, showing the fully activated status with amoeboid shape (Fig. 1f). The perikaya were enlarged, showing coarse and swollen appearance. The processes were shortened and thicken with little branches. The maximum radial extent of these cells (soma axis + longest processes) was less than $30 \mu \mathrm{m}$. In addition, the entire area of the OX42 immunoreactive positive cells including the processes was markedly reduced. Thus, there seems to be a correlation of microglia activative status with neuroprotective effect of LBP. A moderate activation of
Fig. 1 Distribution and morphology of OX42-

immunoreactive microglia in cross-retinal sections. In normal retina (a), only processes without cell body showing OX42- immunoreactive can be detected. In the $\mathrm{OH}$ retina from the PBS feeding group (b), ramified microglia can be detected. In the $\mathrm{OH}$ retina of animals receiving different doses of the Wolfberry extract, $1 \mathrm{mg} / \mathrm{kg}(\mathbf{c}), 10 \mathrm{mg} / \mathrm{kg}$ (d) and $100 \mathrm{mg} / \mathrm{kg}(\mathbf{e})$, there was an increase both in the number and immune intensity of OX42 microglia. In the $1000 \mathrm{mg} / \mathrm{kg}$ group (f), increased number of fully activated microglia was detected. They contained coarse and swollen perikarya that connected with thick processes. Scale bar is $50 \mu \mathrm{m}$

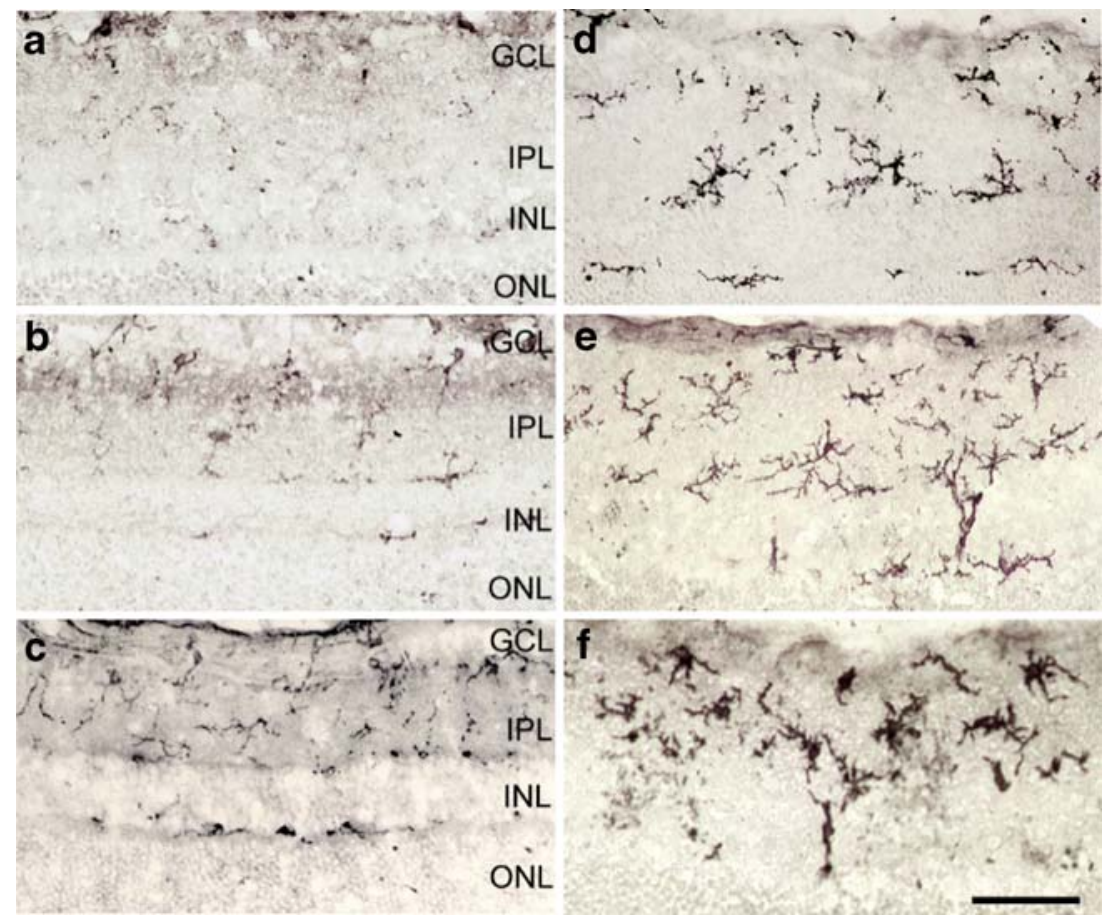


microglia may be correlated with the neuroprotective effect of LBP in $1-100 \mathrm{mg} / \mathrm{kg}$ groups. However, in the $1,000 \mathrm{mg} / \mathrm{kg}$ group, less neuroprotection is linked to fully activated status of microglia.

In order to further illustrate the detailed morphology of the various status of microglia, a multiphoton laserscanning microscope was used to reconstruct the entire microglia on whole-mounted retina. For this purpose, polyclonal ionized calcium adaptive molecular 1 (iba-1) primary antibody was used. The iba-1 protein is specifically localized in microglia and is not found in neurons, astrocytes, or oligodendroglia $[31,32]$. Expression of iba-1 is enhanced when microglia is activated [33]. The retinas were first flat-mounted for counting of RGCs and then refloated to go through the immunohistochemical staining. The morphology of microglia was investigated using multiphoton laser-scanning microscope (LSM-510Meta, Zeiss).

At 4 weeks after the first laser photocoagulation, confocal images by stacking all $\mathrm{Z}$ layers in the inner retina (from the nerve fiber layer to the INL) showed the slightly activated microglia in the PBS-fed group (group 9); they displayed small perikarya and two or more thin branching processes, which were longer than the soma diameter (Fig 2a). In $1 \mathrm{mg} / \mathrm{kg}$ LBP-fed rats, microglia displayed increased iba-1 immunoreactivity in both the soma and processes. There was enlargement of the soma and regional thickening of the processes (Fig 2b). At 4 weeks, LBP significantly reduced the loss of FG-labeled cell from $21.1 \%$ (PBS fed) to $6.6 \%$ (as previously reported [11]). Therefore, consistent with the 2-week observation after administration of LBP in $\mathrm{OH}$ rats, moderately activated microglia were linked to neuroprotection of RGCs in $\mathrm{OH}$ rats.

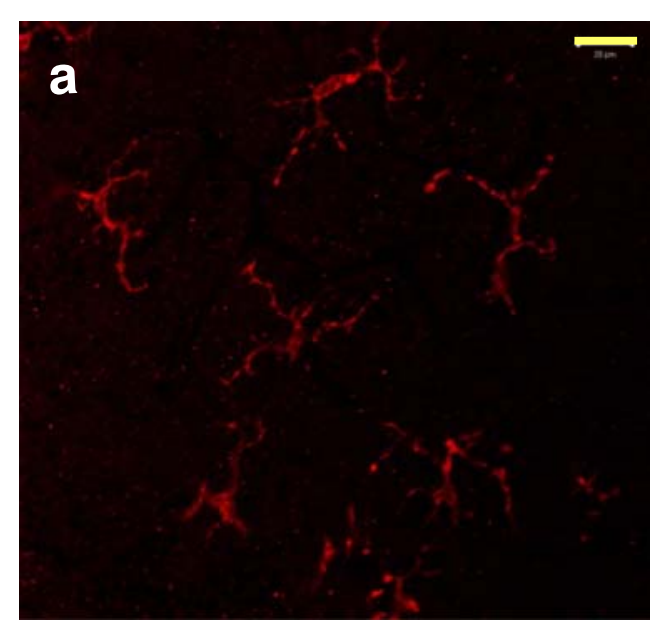

Fig. 2 Morphology of iba-1 immunoreactive microglia in flatmounted retinas at four weeks after the first laser photocoagulation. Confocal images by stacking all $\mathrm{Z}$ layers in the inner retina (from the nerve fiber layer to the inner nuclear layer) showed that morphology of the resting microglia in the PBS-fed group displayed small
Will the neuroprotective effect be affected if microglia are fully activated? To test the effect of fully activated microglia in the retina under $\mathrm{OH}$, bacterial endotoxin LPS was intravitreously injected immediately after the first laser. The effect on the survival of RGCs was observed at 4 weeks time point. Intravitreous injection of LPS significantly increased the loss of RGCs from 21.1 $\pm 1.5 \%$ (PBS fed) to $28.1 \pm 1.9 \%(p<0.05$, Fig. $3 b)$. Neither the neuroprotective effect of LBP nor the neurodestructive effect of LPS on the survival of RGCs was linked to any changes of IOP after the laser treatment (Fig. 3a).

The morphology of one of the representative microglia at resting, moderate, and fully activated status was scanned at $\times 63$ magnification. In normal retina, resting microglia (diameter, $\sim 50 \mu \mathrm{m}$ ) exhibited ramified shape with small nuclei and long thin processes and were located in the inner retina with almost no overlapping of processes (Fig. 4a). At 4 weeks of $\mathrm{OH}$, microglia in the LBP-fed rats (Fig 4b) showed a moderatively activated morphology with increased iba-1 immunoreactivity in the soma and processes. The processes were shortened with focal enlargement compared with resting microglia. Intravitreous injection of LPS at $5 \mu \mathrm{g}$ greatly alerted microglia from a resting state to a fully activated state in the hypertensive eyes; immunoreactivity of iba-1 in the microglia was dramatically increased. They displayed enlarged nuclei and significantly thicker and shorter processes (Fig. 4c).

To test whether the existence of moderately activated microglia have the neuroprotective effects, activation of microglia was attenuated by using intravitreous injection of MIF. Compared with intravitreous injection of PBS $(1.8 \pm$ $2.5 \%)$, there was a greater loss of $\operatorname{RGCs}(9.7 \pm 1.1 \%, p=$ 0.026 ) after the intravitreous injection of MIF (Fig. 5b) in

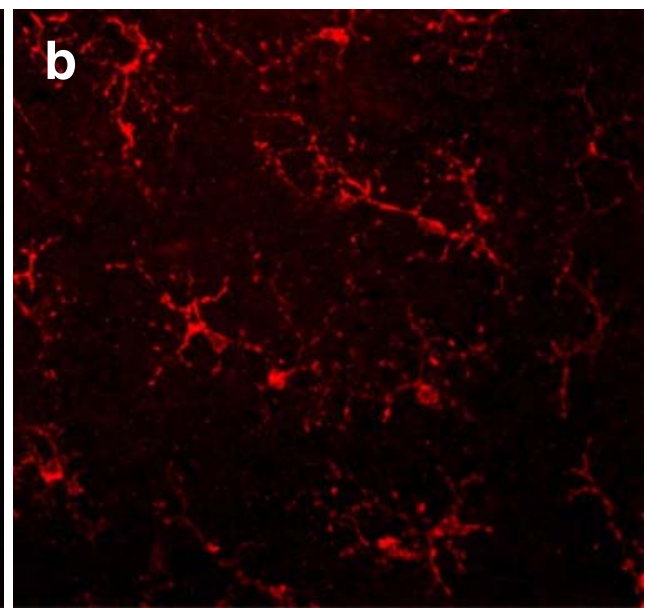

perikarya and two or more thin branching processes, which are longer than the soma diameter (a). In LBP-fed rats, the microglia displayed increased iba-1 immunoreactivity in both the soma and processes. There was enlargement of the soma and regional thickening of the processes (b). Scale bar is $20 \mu \mathrm{m}$ 


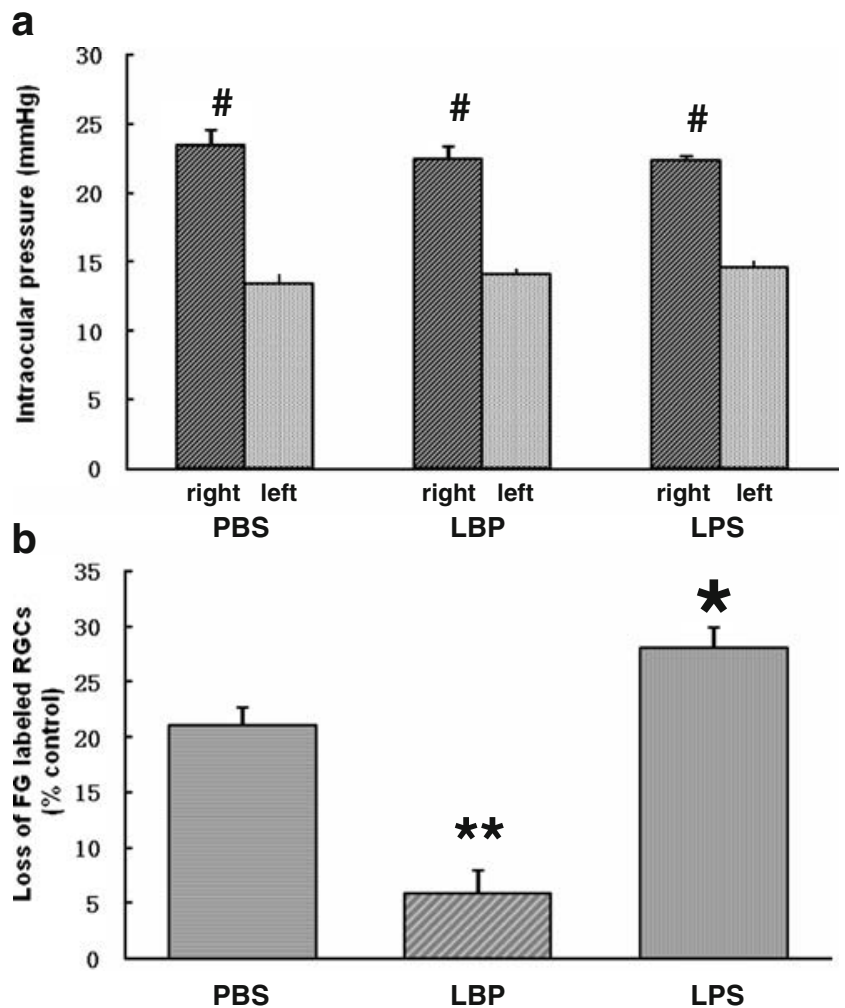

Fig. 3 Profile of the intraocular pressure (IOP) (a) and loss of FGlabeled RGCs in the $\mathrm{OH}$ retinas (b) from PBS, LBP-fed rats or LPS intraocular injection at 4 weeks after the first laser treatment. IOP of the right eye was significantly ${ }^{\#} p<0.05$ ) elevated to about 1.5 -fold to that of the contralateral left control eye (a). There was no significant difference in the eyes among PBS-fed, LBP-fed, and LPS intraocular injection groups. Compared with PBS-fed control (b), $1 \mathrm{mg} / \mathrm{kg} \mathrm{LBP}$ daily feeding significantly reduced the loss of RGCs at four weeks $(* * p<0.001)$, while LPS intraocular injection increased the loss of RGCs $(* p<0.05)$. Error bar represents SEM. The LBP result was reported previously [11] and reproduced here for comparison

the LBP-fed rats. Intravitreous injection of PBS did not affect the survival of RGCs in LBP-fed rats following induction of $\mathrm{OH}$. The elevated IOP was not altered by intravitreous injection of MIF or PBS (Fig. 5a). The results implicated that neuroprotective effects of LBP were partly due to the modulation of the activation of retinal microglia.

\section{Discussion}

Our results suggest that the neuroprotective effects of LBP are partly due to the modulation of activation status of microglia. Concomitant with neuroprotective effect of LBP $(1-100 \mathrm{mg} / \mathrm{kg})$, microglia in the retina are moderately activated. Confocal image of the moderately activated microglia in the inner retina exhibits ramified morphology with thicker and focally enlarged processes, which are different from the resting microglia. This kind of morphology is different to what we can observe by using LPS to elicit fully activated microglia. Appearance of this form of microglia is correlated to the neuroprotection of LBP because the use of MIF to inhibit activation of microglia can attenuate the neuroprotective effect of LBP.

Microglial cells are considered to be resident immune cells in the CNS. In the normal mature brain, "resting" microglia constantly extend their processes, with extention and retraction of the processes as well as motile filopodiumlike protrusions $[34,35]$. Therefore, they are named as "surveillance" microglia because they actively search for and sense any signal in their neighboring environment [36]. Activation of microglia has been considered as a stepwise

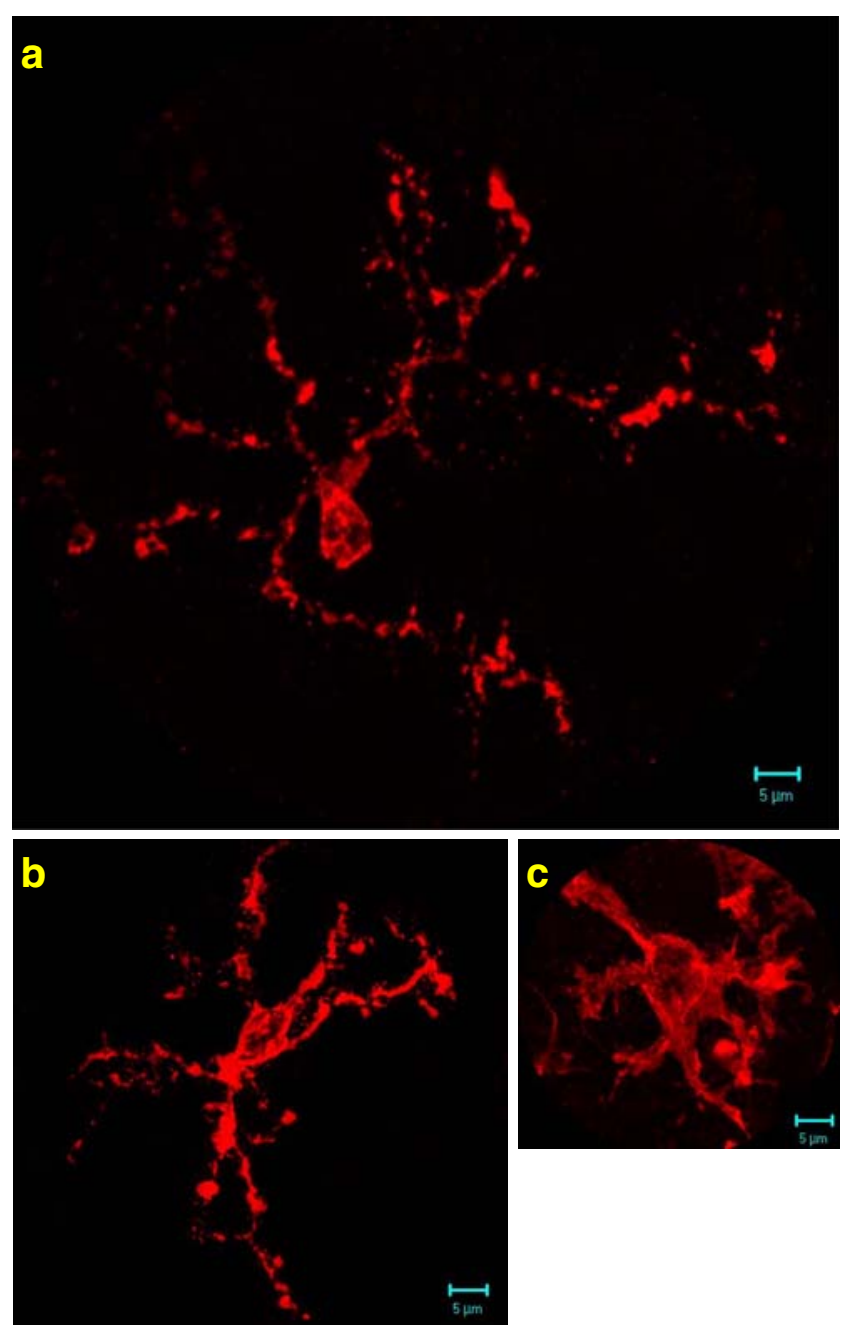

Fig. 4 Z-stacked confocal images in the inner retina showing three types of representative microglial phenotype. In the normal retina (a), the resting microglia showed ramified morphology with small perikarya and three highly branching thin processes. At 4 weeks under the ocular hypertension, microglia in the LBP-fed rats (b) showed increased iba-1 immunoreactivity in the soma and certain enlarged long processes. The processes were shortened compared to resting microglia with focal enlargement. In the $\mathrm{OH}$ retina at 4 weeks following intravitreal injection of LPS (c), microglia was fully activated, showing swollen perikarya and several thick processes with size similar to the soma diameter. Scale bar is $5 \mu \mathrm{m}$ 

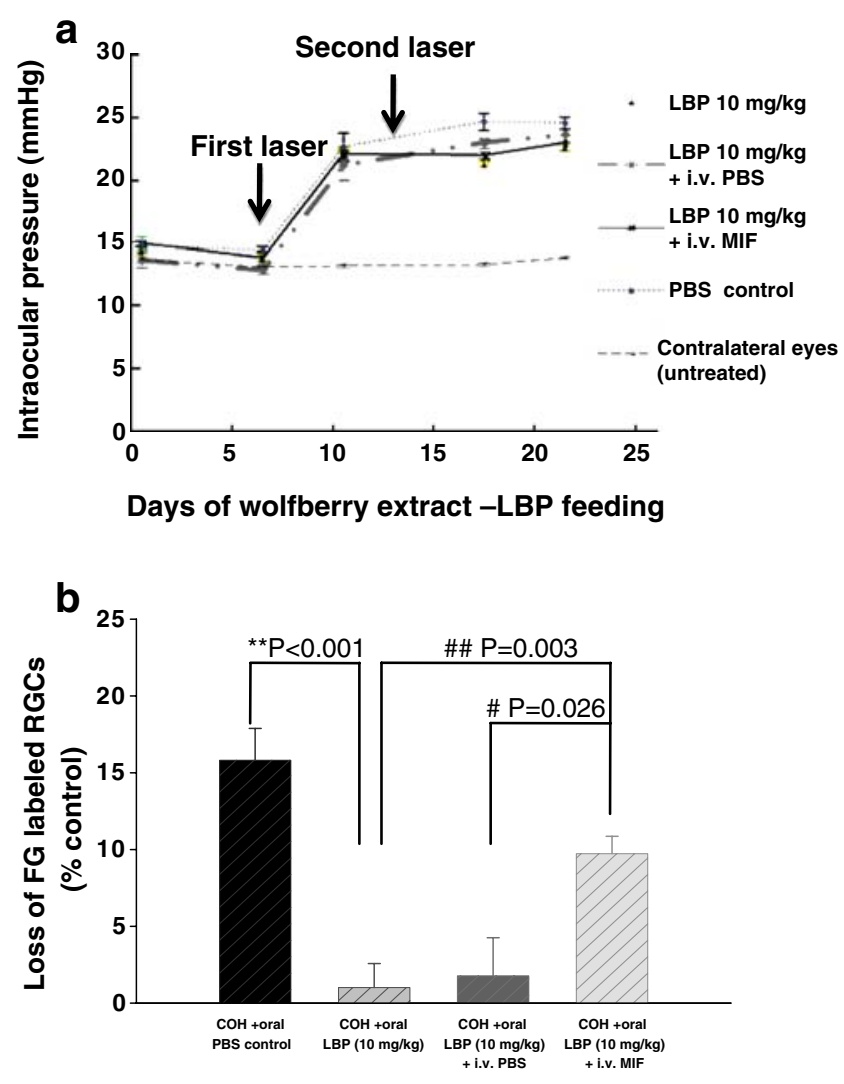

Fig. 5 Profile of the intraocular pressure (IOP) (a) and loss of FGlabeled RGCs in the $\mathrm{OH}$ rat retinas fed with $10 \mathrm{mg} / \mathrm{kg}$ LBP with different intravitreous injections (b). At 2 weeks after the first laser, IOP at the right eyes of all rats was elevated to about 1.5 times to the contralateral control left eyes. There was no difference in the $\mathrm{OH}$ eyes among different groups (a). Neuroprotection from Wolfberry extractLBP on RGCs was not sustained after intravitreous injection of MIF when compared with the intravitreous injection of PBS $\left({ }^{\#} p=0.026\right)$

transformation from resting state (ramified morphology) to activated state (amoeboid morphology) in response to pathological stimuli. However, based on the new findings from in vivo imaging [34, 35], activation of microglia should no longer be considered to be an all or none or onestep event. As pointed out by Hanisch and Kettenmann, the transition between resting and activated states should be considered to be a change in their functions [36]. Activation of microglial cells can result in different morphologies with diverse functions. Engagement of microglia can be either neuroprotective or neurotoxic, resulting in containment or aggravation of disease progression [14, 36]. Our study provides in vivo evidence that a moderately activated microglial morphology correlates with the survival of neurons in ocular hypertensive retina.

The important role of moderately activated microglia was further strengthened by using MIF in our experiment. Both in vitro and in vivo experiments show that MIF directly inhibits the activation of microglia/macrophages [37-39] and has no direct effect on neuron viability [40]. In our experiment, the use of MIF attenuated the neuroprotective effect of LBP. The results suggest that activation of microglia at least partially contributed to the neuroprotective effect of LBP.

LPS has long been considered to be a potent stimulus for microglia. Intravitreous injection of LPS decreases the survival of RGCs at 4 weeks and stimulated microglia to amoeboid morphology, which is consistent with the morphology of activated microglia described previously (Fig. 5c). The increased death of RGCs should not be due to direct neurotoxic effect of LPS, as it has been shown that LPS does not exert direct neurotoxicity on neurons [41]. Therefore, fully activated microglia are neurodestructive rather than neuroprotective.

The modulation of body immunity is often one of the first indicators to access how a Chinese medicine improves our overall body health [42-44]. It has been shown that LBP can increase phagocytic activity of macrophages, antibodies secreted by spleen cells, lymphocyte proliferation in spleen, and activity of cytotoxic T cells [7-9]. In vitro, LBP has been demonstrated to induce maturation of murine bone marrow-derived dendritic cells to secrete IL$12 \mathrm{p} 40$ and increase the expression of membrane markers I-A/I-E and CD11c [45]. This study provides the first in vivo evidence that neuroprotective effects of LBP in rat glaucoma model may be partly due to the modulation of microglia in the retina.

There is also a possibility that LBP provides direct protection of the RGCs against OH. LBP has been shown to improve cognitive functions by enhancing the spontaneous electrical activity of the hippocampus in vivo [46]. In view of the direct cytoprotective and anti-aging effects, it has been shown that LBP counteracts $\beta$-amyloid peptide toxicity in primary neuronal cell culture [26, 47-49]. Instead of anti-oxidation property of LBP [50, 51], it can inhibit two key pro-apoptotic signaling pathways (JNK and PKR) in A $\beta$ peptide neurotoxicity $[47,48,52,53]$. Recently, a new arabino-galactan-protein (LBP-III) isolated from LBP was reported to attenuate the $A \beta$ peptide-triggered caspase-3like activity and the phosphorylation of PKR [26]. Therefore, pro-apoptotic signaling pathways, including PKR, JNK and caspase-3 like activity, should also be evaluated in the LBP neuroprotection against apoptotic RGCs deaths in experimental glaucoma.

According to the theory of Chinese medicine, Wolfberry (L. barbarum) may modulate the energy flow like "Qi" in our body, meaning that it can modulate one organ indirectly by affecting other organs. For example, $10 \mathrm{mg} / \mathrm{kg} \mathrm{LBP}$ can significantly reduce blood glucose, nitric oxide, and malondoaldehyde levels in streptozotocin-induced diabetic rats [54]. Therefore, in our previous study reporting neuroprotective effects of LBP on the survival of RGCs against $\mathrm{OH}$, vital organs were also collected to comprehensively 
study LBP biological mechanisms. We hope to use this experimental glaucoma model as an example to illustrate both the "direct" and "indirect" effects of Wolfberry as proposed by Chang and So [1]; the results can guide us how to make use of Wolfberry for therapeutic intervention of glaucoma in the future.

Acknowledgements This study was supported by the National Glaucoma Research of American Health Assistant Foundation to RCCC, funding from the Jessie Ho Professorship in Neuroscience (The University of Hong Kong Foundation for Educational Development and Research Limited and donation from Mr. George Ho) to KFS, and the Azalea (1972) Endowment Fund to KFS \& RCCC.

Competing interests statement The authors declare that they have no competing financial interests.

Open Access This article is distributed under the terms of the Creative Commons Attribution Noncommercial License which permits any noncommercial use, distribution, and reproduction in any medium, provided the original author(s) and source are credited.

\section{References}

1. Chang RCC, So KF. Use of anti-aging herbal medicine, Lycium barbarum, against aging-associated diseases. What do we know so far? Cell Mol Neurobiol. 2008;28:643-52.

2. Gan L, Zhang S-H, Liu Q, et al. A polysaccharide-protein complex from Lycium barbarum upregulates cytokine expression in human peripheral blood mononuclear cells. Eur J Pharmacol. 2003;471:217-22.

3. Zhou ZW, Zhou JH, Xing ST. Effects of Lyciium barbarum polysaccharides on proliferation and differentiation of hematopoietic stem cells and progenitors of granulocytes and macrophages in mouse bone marrow. Chin J Pharmacol Toxicol. 1991;5:44-6.

4. Du S Y, Zhang X M and Qian Y K (1994) The immunomodulatory effects of lycium barbarum extractions and its mechanism. 3rd International Congress on Ethnopharmacology and It Contemporary Utilization, China Pharmaceutical Association. Beijing C-35

5. Luo Q, Yan J, Zhang SH. Effects of pure and crude Lycium barbarum polysaccharides on immunopharmacology. Zhong Yao Cai. 1999;22:246-9.

6. Peng XM, Qi CH, Tian GY, et al. Physico-chemical properties and bioactivities of a glycoconjugate LbGp5B from Lycium barbarum L. Chin J Chem. 2001;19:842-6.

7. Wang L, Zhao C, Li X, et al. Isolation, purification, analysis and immunity effects of polysaccharide of Lycium barbarum. Acad J Kumming Med Coll. 1995;16:29-32.

8. Wang BK, Xing ST, Zhou H. Effect of Lycium barbarum polysaccharides on the immune responses of T, CTL and NK cells in normal and cyclophosphamide-treated mice. Chin J Pharmacol Toxicol. 1990;4:39-43.

9. Gan L, Hua Zhang S, Liang Yang X, et al. Immunomodulation and antitumor activity by a polysaccharide-protein complex from Lycium barbarum. Int Immunopharmacol. 2004;4:563-9.

10. Hu G, Bai H, Du S. The effects of Chinese medicine Lycium barbarum (LB) on IL-2 production level and IL-2 receptors $(\alpha \&$ ß) expression. Chinese J Immunol. 1995;11:3-256.

11. Chan HC, Chang RCC, Ip AKC, et al. Neuroprotective effects of Lycium barbarum Lynn on protecting retinal ganglion cells in an ocular hypertension model of glaucoma. Exp Neurol. 2007;203: 269-73.

12. Tezel G, Wax MB. Glial modulation of retinal ganglion cell death in glaucoma. J Glaucoma. 2003;12:63-8.

13. Tezel G, Wax MB. The immune system and glaucoma. Curr Opin Ophthalmol. 2004;15:80-4.

14. Schwartz M, Butovsky O, Bruck W, et al. Microglial phenotype: is the commitment reversible? Trends Neurosci. 2006;29:68-74.

15. Lee SC, Liu W, Dickson DW, et al. Cytokine production by human fetal microglia and astrocytes - differential induction by lipopolysaccharide and IL-1-beta. J Immunol. 1993;150:2659-67.

16. Chang RCC, Hudson P, Wilson B, et al. Influence of neurons on lipopolysaccharide-stimulated production of nitric oxide and tumor necrosis factor-[alpha] by cultured glia. Brain Res. 2000; 853:236-44.

17. Chang RCC, Hudson P, Wilson B, et al. Immune modulatory effects of neural cell adhesion molecules on lipopolysaccharideinduced nitric oxide production by cultured glia. Mol Brain Res. 2000;81:197-201.

18. Colton CA, Gilbert DL. Production of superoxide anions by a CNS macrophage, the microglia. FEBS Lett. 1987;223:284-8.

19. Butovsky O, Koronyo-Hamaoui M, Kunis G, et al. From the Cover: Glatiramer acetate fights against Alzheimer's disease by inducing dendritic-like microglia expressing insulin-like growth factor 1. Proc Natl Acad Sci U S A. 2006;103:11784-9.

20. Butovsky O, Landa G, Kunis G, et al. Induction and blockage of oligodendrogenesis by differently activated microglia in an animal model of multiple sclerosis. J Clin Invest. 2006;116: 905-15.

21. Butovsky O, Ziv Y, Schwartz A, et al. Microglia activated by IL-4 or IFN-[gamma] differentially induce neurogenesis and oligodendrogenesis from adult stem/progenitor cells. Mol Cell Neurosci. 2006;31:149-60.

22. Butovsky O, Kunis G, Koronyo-Hamaoui M, et al. Selective ablation of bone marrow-derived dendritic cells increases amyloid plaques in a mouse Alzheimer's disease model. Eur J NeuroSci. 2007;26:413-6.

23. Morgan SC, Taylor DL, Pocock JM. Microglia release activators of neuronal proliferation mediated by activation of mitogenactivated protein kinase, phosphatidylinositol-3-kinase/Akt and delta-Notch signalling cascades. J Neurochem. 2004;90:89-101.

24. Streit WJ. Microglia and neuroprotection: implications for Alzheimer's disease. Brain Res Rev. 2005;48:234-9.

25. Li L, Lu J, Tay SSW, et al. The function of microglia, either neuroprotection or neurotoxicity, is determined by the equilibrium among factors released from activated microglia in vitro. Brain Res. 2007;1159:8-17.

26. Yu MS, Lai CSW, Ho YS, et al. Characterization of the effects of anti-aging medicine Fructus lycii on beta-amyloid peptide neurotoxicity. Int J Mol Med. 2007;20:261-8.

27. Ji JZ, Elyaman W, Yip HK, et al. CNTF promotes survival of retinal ganglion cells after induction of ocular hypertension in rats: the possible involvement of STAT3 pathway. Eur J NeuroSci. 2004;19:265-72.

28. Chiu K, Chang RCC, So KF (2007) Laser induced rat chronic ocular hypertension model. J Vis Exp 10, http://www.jove.com/ index/Details.stps?ID $=549$.

29. Chiu K, Chang RCC, So KF (2007) Intravitreous injection for establishing ocular diseases model. J Vis Exp 8, http://www.jove. com/index/Details.stps?ID=313.

30. Chiu K, Lau WM, Yeung SC et al. (2008) Retrograde labeling of retinal ganglion cells by application of Fluoro-Gold on the surface of superior colliculus. J Vis Exp 16, http://www.jove.com/index/ Details.stp? ID =819.

31. Imai Y, Ibata I, Ito D, et al. A novel gene ibal in the major histocompatibility complex class III region encoding an EF hand 
protein expressed in a monocytic lineage. Biochem Biophys Res Commun. 1996;224:855-62.

32. Ito D, Imai $\mathrm{Y}$, Ohsawa $\mathrm{K}$, et al. Microglia-specific localisation of a novel calcium binding protein, Iba1. Mol Brain Res. 1998;57:1-9.

33. Ito D, Tanaka K, Suzuki S, et al. Enhanced expression of Iba1, ionized calcium-binding adapter molecule 1 , after transient focal cerebral ischemia in rat brain. Stroke. 2001;32:1208-15.

34. Nimmerjahn A, Kirchhoff F, Helmchen F. Resting microglial cells are highly dynamic surveillants of brain parenchyma in vivo. Science. 2005;308:1314-8.

35. Davalos D, Grutzendler J, Yang G, et al. ATP mediates rapid microglial response to local brain injury in vivo. Nat Neurosci. $2005 ; 8: 752-8$.

36. Hanisch UK, Kettenmann H. Microglia: active sensor and versatile effector cells in the normal and pathologic brain. Nat Neurosci. 2007;10:1387-94.

37. Auriault C, Joseph M, Tartar A, et al. Characterization and synthesis of a macrophage inhibitory peptide from the second constant domain of human immunoglobulin G. FEBS Lett. 1983;153:11-5.

38. Raibon E, Sauve Y, Carter DA, et al. Microglial changes accompanying the promotion of retinal ganglion cell axonal regeneration into peripheral nerve grafts. J Neurocytol. 2002;31:57.

39. Thanos S, Mey J, Wild M. Treatment of the adult retina with microglia-suppressing factors retards axotomy-induced neuronal degradation and enhances axonal regeneration in vivo and in vitro. J Neurosci. 1993;13:455-66.

40. Balasingam V, Yong VW. Attenuation of astroglial reactivity by interleukin-10. J Neurosci. 1996;16:2945-55.

41. Bronstein DM, Perez-Otano I, Sun V, et al. Glia-dependent neurotoxicity and neuroprotection in mesencephalic cultures. Brain Res. 1995;704:112-6.

42. Kuroiwa A, Liou S, Yan H, et al. Effect of a traditional Japanese herbal medicine, Hochu-ekki-to (Bu-Zhong-Yi-Qi Tang), on immunity in elderly persons. International Immunopharmacology. 2004:4:317-24.

43. Xu RR, Cao F, Liu ZX. Clinical observation on treatment of acute myelocytic leukemia by supplementing qi, nourishing yin and clearing heat principle. ZhongGuo Zhong Xi Yi Jie He Za Zhi. 2004;24:411-4.
44. Xiao PG, Liu CX. Immunostimulants in traditional Chinese Medicine. In: Wagner H, editor. Immunomodulatory Agents from Plants. Basel/Switzerland: Birkhäuser Verlag; 1999. pp. 325-356.

45. Zhu J, Zhao L-H, Zhao X-P, et al. Lycium barbarum polysaccharides regulate phenotypic and functional maturation of murine dendritic cells. Cell Biol Int. 2007;31:615-9.

46. Peng XD, Xiu DQ, Peng JZ, et al. Effects of Lycium barbarum polysaccharide on hippocampal activity in animals. J Ningxia Med Coll. 2002;24:79-81.

47. Yu MS, Leung SKY, Lai SW, et al. Neuroprotective effects of anti-aging oriental medicine Lycium barbarum against [beta]amyloid peptide neurotoxicity. Exp Gerontol. 2005;40:716-27.

48. Yu MS, Suen KC, Kwok NS, et al. Beta-amyloid peptides induces neuronal apoptosis via a mechanism independent of unfolded protein responses. Apoptosis. 2006;11:687-700.

49. Ho YS, Yu MS, Lai CSW, et al. Characterizing the neuroprotective effects of alkaline extract of Lycium barbarum on [beta]-amyloid peptide neurotoxicity. Brain Res. 2007;1158: 123-34.

50. Li XM. Protective effect of Lycium barbarum polysaccharides on streptozotocin-induced oxidative stress in rats. Int J Biol Macromol. 2007;40:461-5.

51. Wang ZY, Huang XR, Qi MX. The regulation of LBP (lycium barbarum polysaccharide, LBP) on the expression of apoptosisrelated genes Bcl-2 and Bax in SD rat LEC (lens epithelial cells, LEC) induced by oxidative injuries. Chinese J Optomet Ophthalmol. 2003;5:147-9.

52. Chang RCC, Suen $\mathrm{KC}$, Ma CH, et al. Involvement of doublestranded RNA-dependent protein kinase and phosphorylation of eukaryotic initiation factor-2 alpha in neuronal degeneration. J Neurochem. 2002;83:1215-25.

53. Suen KC, Yu MS, So KF, et al. Upstream signaling pathways leading to the activation of double-stranded rna-dependent serine/ threonine protein kinase in $\{$ beta $\}$-amyloid peptide neurotoxicity. J Biol Chem. 2003;278:49819-27.

54. Wu H, Guo H, Zhao R. Effects of Lysium barbarum polysaccharide on the improvement of antioxidant ability and DNA damage in NIDDM rats. Yakugaku Zasshi J Pharm Soc Jpn. 2006;126: $365-71$.

\section{㸺 Humana Press}

